\title{
Comparative Costs of Different Renal Replacement Therapies in Lower Middle Income Countries on the Example of Georgia
}

\author{
Avtandil Tataradze ${ }^{1}$, George Managadze ${ }^{2}$, Lela Beglarashvili' ${ }^{1}$, Nikoloz Kipshidze ${ }^{3}$, \\ Laurent Managadze ${ }^{1}$, Archil Chkhotua ${ }^{1}$ \\ ${ }^{1}$ Tsulukidze National Center of Urology, Tbilisi, Georgia \\ ${ }^{2}$ Tulane University, New Orleans, USA \\ ${ }^{3}$ Kipshidze Central University Clinic, Tbilisi, Georgia \\ Email: tataradze@dnc.ge, achkhotua@gmail.com
}

Received 18 May 2016; accepted 2 July 2016; published 5 July 2016

Copyright (C) 2016 by authors and Scientific Research Publishing Inc. This work is licensed under the Creative Commons Attribution International License (CC BY). http://creativecommons.org/licenses/by/4.0/

(c) (i) Open Access

\begin{abstract}
End-Stage Renal Disease (ESRD) represents one of the most challenging social and medical problems mainly due to substantial treatment-associated costs. The chronic nature of the disease needs expensive continuous care that majority of the patients cannot afford. Therefore, in many countries expenses associated with the ESRD treatment is paid by state government. These treatment options include: hemodialysis, peritoneal dialysis and kidney transplantation. Multiple studies have been conducted throughout the world to assess cost-effectiveness of these treatment modalities. The studies suggest that kidney transplantation not only reduces mortality and morbidity but improves a quality of life of ESRD patients. Furthermore, it is the most cost-effective treatment for the ESRD at least in high-income countries. The goal of our study was to determine whether above-mentioned is true for lower middle income countries, where the cost of the ESRD treatment is substantially lower. Despite the low dialysis costs, transplantation remains the cheapest form of renal replacement therapy RRT in lower income countries like Georgia. Our results reveal, that kidney transplantation is most expensive modality of Renal Replacement Therapy (RRT) at month 1, but count of costs reveals that after the $10^{\text {th }}$ month of treatment, the cumulative cost of transplantation is less than the cumulative cost of peritoneal dialysis and after the $23^{\text {rd }}$ month, cumulative cost of hemodialysis also surpasses the cumulative cost of transplantationrelated treatment and this cost comparison is in line with global data from upper-middle and high income countries.
\end{abstract}


Keywords

End-Stage Renal Disease (ESRD), Renal Replacement Therapy (RRT) Costs, Hemodialysis,
Peritoneal Dialysis, Kidney Transplantation (KT)

\section{Introduction}

Worldwide, End-Stage Renal Disease (ESRD) represents one of the most challenging social and medical problems due to substantial treatment-associated costs. The chronic nature of the disease requires expensive continuous care that majority of the patients cannot afford. Therefore, in many countries expenses associated with the ESRD treatment is paid by state government. The main modalities of renal replacement therapy include: hemodialysis, peritoneal dialysis and kidney transplantation. Governments, mostly in developed countries, each year face increase of total ESTD treatment costs due the surge of disease incidence and prevalence fed by the factors as aging population trend and raise of diabetes morbidity. Therefore, searching for most efficient ways of treatment is highly demanded globally. Multiple studies have been conducted throughout the world to assess costeffectiveness of these treatment modalities. These studies suggest that kidney transplantation not only reduces mortality and morbidity but improves a quality of life of ESRD patients. Furthermore, it is the most cost-effective treatment for the ESRD at least in upper-middle and high income countries. The goal of our study was to determine whether above-mentioned is also true for lower-middle income countries, where the overall costs of the ESRD treatment is substantially lower. Also we aim to suggest policy improvements that would promote costeffective methods of treatment, which will help relatively low-income Governments such as of Georgia to make available better possible treatment services to the greatest possible number of people with ESRD.

\subsection{Global Picture of ESRD}

Constantly rising number of ESRD patients on all continents drive the global cost of ESRD treatment. In the UK, the annual incidence of ESRD has doubled during 1995-2005 to reach about 100 new patients per million of population. The annual incidence of people older than 65 years in the USA is more than 1200 per million of population [1]. There are two factors that augment the increasing number of ESRD patients. First is the ageing of the population; the incidence of ESRD is higher in elderly people than in general population. The second factor is the global epidemic of type 2 diabetes mellitus. Patients with diabetes mellitus have higher chances of developing ESRD [2]. In 2009, costs of ESRD rose by 3.1\% to 29\% billion within US [3].

It is doubtless that higher incidence rates of ESRD patients will result in ever-growing expenditures. There are several ways to avoid ever-growing costs of ESRD treatment. In some countries, hemodialysis can be replaced by cheaper peritoneal dialysis as a main modality of treating ESRD. Moreover, a shift from in-center hemodialysis to home-based dialysis is another potential strategy that could enhance the cost-effectiveness of ESRD care. Overall, studies from various upper-middle to high income countries demonstrate that kidney transplantations is the most cost-effective way to treat ESRD, resulting in reduced expenditures that could be used more productively elsewhere.

The severity of prevalence and incidence of End-Stage Renal Disease is even more alarming in developing countries [4]. Despite the similar incidence rates, the prevalence rates vary from less than 100 per million population in sub-Saharan Africa and India to about 400 per million population in Latin America and more than 600 per million population in Saudi Arabia4. Thus, prevalence is largely a matter of survival made possible by renal replacement therapy, which in turn depends on healthcare expenditures and economic strength of these countries/continents.

\subsection{End-Stage Renal Disease Financing Options}

Various countries have different financing options for End-Stage Renal Disease. A study by Dor, Pauly, Eichleay and Held provides comprehensive review of several financing models that countries participating in ISHCOF (International Study of Health Care Organization and Financing) use. These countries include Australia, Belgium, Canada, France, Germany, Italy, Japan, New Zealand, Spain, Sweden, United Kingdom and United 
States. Health care systems in these countries appear to be markedly different, ranging from the relatively market-based system in the United States to the National Health Service models that have the government as the sole owner and payer for health care (United Kingdom, Sweden, Spain, and Italy).

Three types of models tend to dominate payments to dialysis centers. The first model uses per-treatment prices that are administratively set at a national level. In the second model, payment systems may be based on capitation, i.e., a fixed payment per patient or episode of care. The third model is global budgeting, whereby a regional administrative authority or a major hospital at the head of a local network is responsible for allocating an overall budget to various activities and units under its administrative control [5].

In contrast to the often-complex payment rules for dialysis providers, payments for kidney transplantation tend to be simple and straightforward. For the most part, transplant costs are paid fully by the relevant national health authority [5].

\subsection{Comparative Cost-Effectiveness of ESRD Treatment Options}

Multiple studies have been conducted throughout the world exploring the cost-effectiveness of End-Stage Renal Disease treatment options. These studies unanimously suggest that kidney transplantation is the most cost-effective treatment method for ESRD. Below we will review cost-effectiveness of RRT options in high- and upper middle income countries. Thereafter we will review Georgia, as an example of lower middle income country for the comparative cost-effectiveness of renal replacement therapy options.

Austria-A study conducted in Austria developed a Markov model of costs, quality of life and survival to compare three different assignment strategies of Renal Replacement Therapy. The authors used three strategies to construct their model. Strategy 1 represents the current assignment policy in Austria. 90.6\% of new ESRD patients were treated with hemodialysis, $7.2 \%$ with peritoneal dialysis, $0.1 \%$ received a renal transplantation from a live donor and $2.1 \%$ from a deceased donor. The hypothetical alternative Strategy 2 was set as $20 \%$ of the incident, ESRD patients were allocated to peritoneal dialysis. In the other alternative Strategy 3, 20\% of incident ESRD patients were allocated to peritoneal dialysis and additional $10 \%$ for preemptive renal transplant from a living donor (see Table 1) [6].

Overall, cost-effectiveness study showed that kidney transplantation and peritoneal dialysis perform better when compared to hemodialysis. Strategy 2 (20\% peritoneal dialysis) and Strategy 3 (20\% peritoneal dialysis and 10\% kidney transplantation) can save €26 million and €38 million discounted respectively and gain 839 QALYs and 2242 QALYs respectively over the next 10 years when compared to Strategy 1 (hemodialysis dominated) [6].

Denmark-Similar study in Denmark also used decision analytic tree of Markov model and established the cost-utility analysis (CUA). The analysis was conducted based on data acquired in Denmark from 2012. At the end of year 2012, 4829 patients were being treated for end-stage renal disease (ESRD) in Denmark. Of these, 2330 patients had received kidney transplantation, whereas the remaining 2499 patients were in dialysis. Instead of US dollars, however, the analysis was built on Danish Crones [7].

The CUA shows that when comparing the total average costs and effects of the two alternative treatments, transplantation holds a dominant position as it yields both lower costs (810,516 DKK versus 1,032,934 DKK) and higher effects (4.4 QALY versus 1.7 QALY). Thus, the cost-utility analysis showed that transplantation is more cost-effective compared with dialysis; it yields both lower costs and better effects [7].

Serbia - A study conducted in Serbia aimed to compare ratio of costs and effects (Cost Effectiveness Analysis-CEA) of hemodialysis and kidney transplantation in patients with End-Stage Renal Disease. The study included 150 patients totally, divided into two groups. The study group consisted of 50 patients with kidney transplantation and the control group consisted of 100 patients on hemodialysis. There was no statistically significant age difference between the dialyzed (in average 42.92 years) and transplant (in average 40.58 years) patients ( $p$ $=0.154$, $\mathrm{t}$ test $)[8]$.

An effect of kidney transplantation in relation to hemodialysis as treatment selection was expressed in the form of Incremental Cost-Effectiveness Ratio (ICER), according to the equation: ICER $=$ CTR - CHD/ETR $\mathrm{EHD}=\Delta \mathrm{C} / \Delta \mathrm{E}$, (where CTR - costs of transplant patients for a 10-year period, CHD-cost of patients on hemodialysis for a 10-year period, ETR-QALY years of life for transplant patients, EHD-QALY years of life for patients on hemodialysis).

According to the equation for calculating ICER the costs of kidney transplantation and patient maintenance therapy are considerably lower than costs of hemodialysis within a 10 -year period, as well as that the difference 
Table 1. Four calculated outcomes for three different treatment strategies.

\begin{tabular}{ccccc}
\hline \multirow{2}{*}{ Treatment Strategies } & \multicolumn{3}{c}{ Treatment Outcomes } \\
\cline { 2 - 5 } & Discounted Costs & Total Life Years Saved & Total Years Free of Dialysis & Discounted QALY Years \\
\hline Strategy 1 & $€ 8083 \mathrm{M}$ & 259,731 & 103,387 & 203,407 \\
Strategy 2 & $€ 8057 \mathrm{M}$ & 260,435 & 103,875 & 204,245 \\
Strategy 3 & $€ 8046 \mathrm{M}$ & 261,511 & 107,157 & 205,648 \\
\hline
\end{tabular}

in patient life quality is in favor of patients with kidney transplantation 8. Overall the study concluded that kidney transplantation strategy is far more cost-effective since it saves EUR 132256.25 per one year of QALY contribution within 10 years period [8].

Spain-A prospective study conducted in Hospital Clínic de Barcelona compared the costs of Live -Donor Kidney Transplantation (LDKT) during the first year of the treatment with those of hemodialysis during the first year. Total of 106 patients (57 undergoing HD and 49 who receiving LDKT) participated in the study 9. The mean age of transplant recipients was $46 \pm 15$ years and that of donors was $52 \pm 10$ years. The study found that the mean annual cost of LDKT in the first year after transplantation was 29,897.91€. Of this sum, 27\% (8128.44€) amounted to costs associated to donor. The rest of the 73\% (21,769.47€) represented costs associated to kidney transplant recipient. The cost of hemodialysis was $43000.88 €$, of which $37,917 \mathrm{~V}$ corresponded to HD procedure and activities and the remainder (5082€) to transportation. Overall, the study concluded that live donor kidney transplantation is more cost-effective than hemodialysis, representing a savings of 13,102.97 per patient/y. The study further reported two findings; a. the costs of kidney transplantation shrink after year one, while the costs of hemodialysis remain the same, b. kidney transplantation is associated with higher quality of life (QALY) than hemodialysis treatment, although the study did not report any numerical values associated with QALY [9].

Australia-A study conducted in Australia used a dynamic population-based Markov model to assess the costs and health outcomes of proposed changes in service provision. Specifically, from the viewpoint of a central healthcare funder, study assessed the incremental health sector costs and benefits of i) an increase in the number of new ESKD patients receiving a transplant and ii) increasing the number of new dialysis patients receiving home-based, rather than hospital-based services, compared with current clinical practice for treating people with ESKD. These two proposed changes to clinical practice for new ESRD patients in Australia were assessed over 2005-2010 10. Overall the study concluded that increasing number of kidney transplantations has tri-fold effect; it reduces the costs, improves patient's quality of life (QALY) and increases the patient survival rate. The study also made favorable conclusions towards the home based hemodialysis - that it can produce net savings of \$A46.6 million by 2010 without affecting the quality of treatment [10].

Chile-A study conducted in Chile used the Markov model to assess the net benefits of cadaveric kidney transplantation within the country11. The study found that the expected present value of costs associated with dialysis is approximatelyUS $\$ 134,000$, whereas the expected costs of a transplant amount to nearly US $\$ 106,000$. Overall, the study concluded that cost savings associated with cadaveric kidney donation are positive and substantial, thus cadaveric kidney transplantation is highly encouraged as a primary strategy of renal replacement therapy [11].

Portugal—A retrospective study conducted in Portugal analyzed cost-effectiveness of kidney transplantation, hemodialysis and peritoneal dialysis 7 . The study found that at a 10 year (estimated graft average survival) interval, kidney transplantation costs 120,395.02€ and dialysis 278,091.12€. Thus, over the span of 10 years, renal transplantation saves the Portuguese health system an average of 157,696.1€. Thus, the study concluded that since the renal transplantation is the most cost-effective treatment of End-Stage Renal Disease, the Portuguese government should shift its attention and resources on promoting more organ donation [12].

Italy-A study conducted in Italy assessed the costs and health outcomes of proposed changes in service provision for ESRD patients. Specifically, authors assessed the incremental health care costs and benefits of the following: 1) an increase in the number of new ESRD patients receiving atransplant from donation after cardiac death (DCD); and 2) an increase in the total number of transplants (all types) supplied to new ESRD patients, compared with current clinical practice for treating patients with ESRD in the same setting [13]. The study was based on a dynamic population-based Markov, estimating the costs and benefits of proposed changes in service delivery to ESRD patients. Overall, the study concludes that changing the actual practice pattern for new patients 
with ESRD by increasing the availability of kidneys from DCD should result in a cost-effective policy to expand the kidney donor pool. Thus, the authors argue that their results should encourage a health policy expressively tailored to reach markedly higher rates of kidney transplants by using extended criteria to select donors such as those experiencing cardiac death [13].

The eight studies above argue that kidney transplantation represents the most cost effective treatment option for End-Stage Renal Disease compared to hemodialysis and peritoneal dialysis. This opinion based on evidence is now widely shared among the countries of different development level including medium and high income countries. However, universality of this principle is yet to be tested also in lower-income countries. In this paper we will try to examine and compare RRT cost in Georgia, which is lower-middle income country based on World Bank ranking.

\section{Methods and Results}

\subsection{ESRD Treatment in Georgia}

Georgia, being a small post-soviet country is regarded as a lower middle income economy. The current funding model for ESRD treatment has been inaugurated in 2011 as a result of a wide reform, which amid at universal and accessible medical care for all patients with ESRD. The prevalence of dialysis-dependent patients in Georgia is about 570 per million populations and this number is progressively increasing due to factors such as increased access to treatment and overall increase in life expectancy.

For instance, the total number of beneficiaries of the state hemodialysis program has increased by $16 \%$ and $11 \%$ in years 2014 and 2015, respectively. These figures reflect a substantial growth in burden of costs as well.

Georgia is a good example for comparing costs associated to ESRD treatment in a lower income country. As the state is providing funds for all three modalities of Renal Replacement Therapies, we are able to perform detailed analysis of the cost-structure associated to each of those. The goal of this paper is to provide evidence that along with upper-middle and high income countries such as above reviewed Serbia and USA, Kidney Transplantation is the most cost-effective method of RRT also in lower income countries, which have a different cost structure and expectedly lower per patient expenditure compared to richer countries.

Although public funding is ensured for each of RRT, the allocation and drivers of financing varies across the modalities because of legal and other limitations, which will be discussed further in this paper especially with regard to kidney transplantation.

\subsection{Hemodialysis in Georgia}

State provides $100 \%$ funding for hemodialysis treatment in Georgia, which covers dialysis sessions, basic lab tests and medications and renal anemia therapy (Erythorpoeitin and IV iron) and the services are immediately available for all patients diagnosed with ESRD. The principle of funding is per dialysis session per patient and covers up to 157 hemodialysis procedures per year (based on frequency of three sessions per week) and the total funding consists of two different parts:

1) Reimbursement of service per hemodialysis sessions; and

2) Centralized state purchase of the dialysis disposables and anti-anemic medications;

For the reimbursement of dialysis the state currently pays GEL 40.0 (US\$ 18) per hemodialysis session to service provider directly, which should cover the cost of dialysis service, including salaries, management, equipment as well as lab tests and medications (usually limited to basic tests and generic antihypertensives, phosphate binders and antibiotics). For the medication and other materials needed for hemodialysis government centrally makes purchases and makes allocations to service providers based on request as per usage incurred. Centrally purchased medications comprise anticoagulants and anti-anemic drugs (generic erythropoietin and generic iron sucrose), as well as dialysis disposables, which typically include dialyzer, dialysis blood set, needles, bicarbonate, acid concentrate and disinfectant. We have calculated the cost of centralized state purchase per patient per hemodialysis session based on the allocations made during last 12 months to National Centre for Urology—a major ESRD treatment provider in Georgia, which handles approx. 10\% of country's total yearly hemodialysis budget and 50\% of peritoneal dialysis and 50\% of Kidney Transplantations in Georgia. National Centre for Urology data from last 12 months (April 2015 to March 2016) suggest that Government allocated on average GEL 44 (US\$ 20) worth of centrally purchased medications and materials per hemodialysis session per 
patient. Combining the funding of dialysis sessions (GEL 40) and allocated medications and materials (GEL 44) amounts to GEL 84 (US\$ 37) total hemodialysis budget per session per patient, which multiplied by the reimbursed number of hemodialysis sessions entitled to a patient results in GEL 13,236 (US\$ 5883) average hemodialysis budget Per Patient Per Year (PPPY) as of year 2016.

\subsection{Peritoneal Dialysis in Georgia}

Public funding of peritoneal dialysis in Georgia also consists of two parts:

1) Reimbursement to clinics providing peritoneal dialysis_-amount defined per patient per month; and

2) Centralized state purchase of the dialysis disposables and anti-anemic medications;

Fixed funding which state directly pays to service providers amounts to GEL 105.0 (US\$ 47) per patient per month. For the medication and other materials needed for peritoneal dialysis government centrally makes purchases and makes allocations to service providers based on request as per usage incurred. National Centre for Urology data from last 12 months (April 2015 to March 2016) suggest that Government allocated on average GEL 2115 (US\$ 940) worth of centrally purchased medications and materials per peritoneal dialysis patient per month, which together with monthly fixed reimbursement per patient amounts to GEL 26,639 (US\$11,840) average peritoneal dialysis budget Per Patient Per Year (PPPY) as of year 2016. Thus, the cost of peritoneal dialysis in Georgia is double of hemodialysis making it significantly inefficient from the costs point of view and how this circumstance affects access to peritoneal dialysis is subject to further analysis.

As displayed on Table 2, peritoneal dialysis in Georgia is at least double the cost of hemodialysis, whereas in Austria, the ratio is exactly the opposite, hemodialysis being significantly more expensive. This is to be attributed to difference in the cost structure associated with these treatment methods. Hemodialysis has been observed as a labor-intensive treatment, with salaries taking significant part of the cost, whereas the biggest contributor to peritoneal dialysis expense is its material [14]. NayakKaropadi et al. have compared the costs of hemodialysis and peritoneal dialysis in 46 counties (20 developed and 26 developing) and found out that hemodialysis to peritoneal dialysis ratio tends to be higher in developed countries, implying high labor costs [15]. In Georgia low salaries drive the low cost of hemodialysis, while the cost of peritoneal dialysis stands on par with global prices of its prime materials.

\subsection{Kidney Transplantation in Georgia}

Kidney transplantation is the third method of Renal Replacement Therapy in Georgia, which is publicly funded, however the number of cases is limited by available funds to the maximum of 36 surgeries per year.

Its mechanism of financing also consists of two parts:

1) Reimbursement of surgery and early post transplatation period; and

2) Centralized state purchase of immunosuppressive medications;

Government directly pays to service providers fixed amount of GEL 20,000 (US\$8.889) per kidney transplantation surgery, and provides patients with centrally purchased very basic immunosuppressive medications, limited to generics of CNI (both Cyclosporin and Tacrolimus are used) lifelong and MMF only for the first 6 post-transplant months (lifelong only in selected cases), with no induction therapy or alternative drugs covered. Based on data from the National Centre for Urology, the average cost of immunosuppressive medications during firs 6 post-transplant months amounts to GEL 240 (US\$ 107) per month. After the $7^{\text {th }}$ moth the average cost per patient per month decreases to GEL180 (US\$ 80).

As we want to compare economics of dialysis and kidney transplantation as alternative methods of Renal

Table 2. Comparison of TTP costs in three different income level countries.

\begin{tabular}{|c|c|c|c|c|c|}
\hline Year & Country & Income Level & HD Cost PPPY (EUR) & PD Cost PPPY (EUR) & KT Cost PPPY (EUR) \\
\hline 2008 & Serbia & Upper-middle & 10 year average $-16,533$ & N/A & 10 year average $\_4895$ \\
\hline 2010 & Austria & High & $\begin{array}{c}1^{\text {st }} \text { year }-43,600 \\
2^{\text {nd }} \text { year }-40,000 \\
>2^{\text {nd }} \text { year }-40,600\end{array}$ & $\begin{array}{c}1^{\text {st }} \text { year }-25,900 \\
2^{\text {nd }} \text { year }-15,300 \\
>2^{\text {nd }} \text { year }-20,500\end{array}$ & $\begin{array}{c}1^{\text {st }} \text { year }-51,000 \\
2^{\text {nd }} \text { year }-17,200 \\
>2^{\text {nd }} \text { year }-12,900\end{array}$ \\
\hline 2016 & Georgia & Lower-middle & Yearly-6765 & Yearly-13,600 & $\begin{array}{c}1^{\text {st }} \text { year }-12,670 \\
\text { From } 2^{\text {nd }} \text { year }-1835\end{array}$ \\
\hline
\end{tabular}


Replacement Therapies, we put those costs on a single chart to review the cost-effectiveness over years of treatment (see Figure 1).

As we can observe from the Figure 1, kidney transplantation is most expensive modality of RRT at month 1, but count of costs reveals that after the $10^{\text {th }}$ month of treatment, the cumulative cost of transplantation is less than the cumulative cost of peritoneal dialysis and after the $23^{\text {rd }}$ month, cumulative cost of hemodialysis also surpasses the cumulative cost of transplantation-related treatment and this cost comparison is in line with global data from upper-middle and high income countries as we reviewed above (Table 2). This effect is possible because after initial high cost of surgery and intensive immunosuppressive engagement, the monthly cost of treatment from the seventh month drops to just $16 \%$ and $8 \%$ of monthly costs of peritoneal dialysis and hemodialysis, respectively.

However, despite being the most cost-effective method of treatment for ESRD, kidney transplantation is paradoxically the only financially restricted RRT in Georgia, as currently the state makes available funds for maximum of 36 surgeries per year. Other objective restrictions to kidney transplantation include legal framework on organ donation in Georgia, which only allows donations from living relatives, including parents, siblings, spouse, children and some other close relatives. Dating back from grim past of warfare, criminal and economic hardship after the collapse of the Soviet Union, Georgia enacted strict donor laws, forbidding not legally related donor transplantation to avoid illegal market for organ trade. Nonexistence of state funded potential donor examination programs farther discourages kidney transplantation.

\section{Recommendations to Promote Kidney Transplantation in Georgia}

As demonstrated by the case of Georgia, transplantation represents the cheapest form of RRT also in lower income countries. This is a good financial argument for the Georgian government to try to maximize the number of kidney transplantations and reduce the number of patients on maintenance dialysis. Following measures can help promote kidney transplantation as the method of choice of RRT:

1) Removing financial obstacles and providing universal coverage for kidney transplant surgeries without delay, much as in case of hemo- and peritoneal dialysis. As funds are restricted only for kidney transplantations, it is creating the barrier to accessibility. Removing the limit of yearly kidney transplantations would increase RRT cost in the first year, which would be offset by significantly lower post-transplantation medication costs;

2) Providing funds for potential living donor examinations would help increase the number of successful kidney transplantations by reducing health risks and thus attracting more living donors;

3) Expanding the potential donors' circle by removing legal obstacles and promoting altruistic living donations to friends and other emotionally close but not legally related patients. This proposal is currently under active consideration and could potentially double the number of living donors for an average patient;

4) Improving post-transplant care coverage by providing better and more modern immunosuppression, comprehensive post-transplant surveillance and treatment programs for complications;

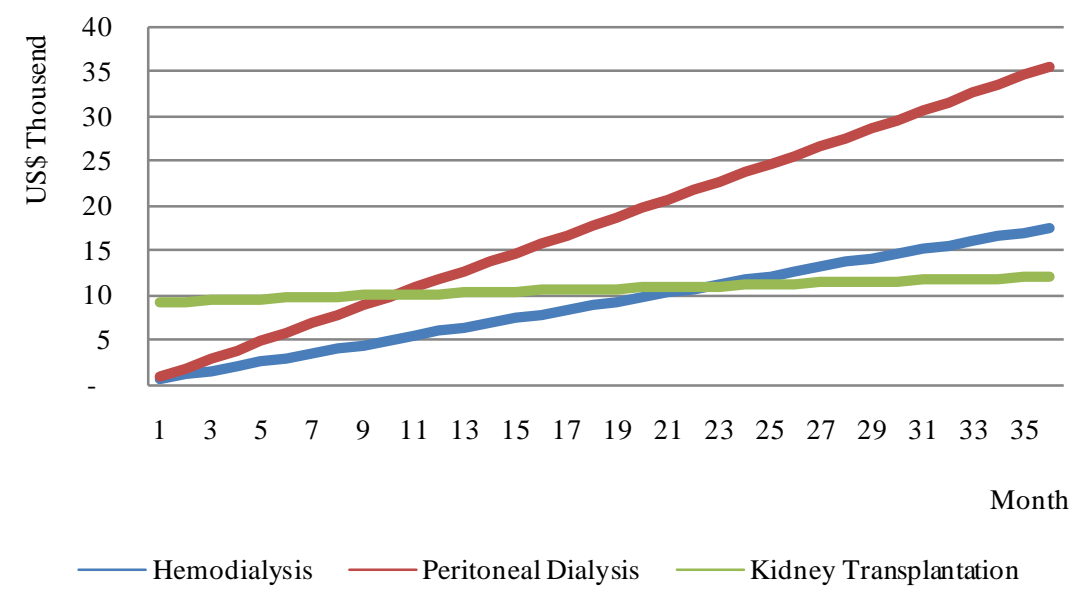

Figure 1. Cumulative cost of treatment, US\$. 
5) Taking reasonable initial steps to introduce a state program of deceased donor transplantations. Studies 6 have shown that live donor and deceased donor kidney transplantations have comparable effects on the patients in terms of cost-effectiveness, graft viability and survival rates. Georgia could start preparations involving the work on public opinion, infrastructure and legal frameworks to put a basis for future decease donor transplantations.

\section{References}

[1] End Stage Renal Disease in the United States. National Kidney Foundation.

[2] Meguid El Nahas, A and Bello, A.K. (2005) Chronic Kidney Disease: The Global Challenge. The Lancet, 365, 331-340. http://dx.doi.org/10.1016/S0140-6736(05)17789-7

[3] USRDS Annual Data Report (2011) Costs of ESRD.

[4] Barsoum, R.S. (2006) Chronic Kidney Disease in the Developing World. The New England Journal of Medicine, 354, 997-999. http://dx.doi.org/10.1056/NEJMp058318

[5] Dor, A., Pauly, M.V., Eichleay, M.A. and Held, P.J. (2007) End-Stage Renal Disease and Economic Incentives: The International Study of Health Care Organization and Financing (ISHCOF). International Journal of Health Care Finance and Economics, 7, 73-111. http://dx.doi.org/10.1007/s10754-007-9024-9

[6] Haller, M., Gutjahr, G., Kramar, R., Harnoncourt, F. and Oberbauer, R. (2011) Cost-Effectiveness Analysis of Renal Replacement Therapy in Austria. Nephrology Dialysis Transplantation, 26, 2988-2995. http://dx.doi.org/10.1093/ndt/gfq780

[7] Jensen, C.E., Sørensen, P. and Petersen, K.D. (2014) In Denmark Kidney Transplantation Is More Cost-Effective than Dialysis. Danish Medical Journal, 61, A4796.

[8] Perović, S. and Janković, S. (2009) Renal Transplantation vs Hemodialysis: Cost-Effectiveness Analysis. Vojnosanitetski Pregled, 66, 639-644. http://dx.doi.org/10.2298/VSP0908639P

[9] Sánchez-Escuredo, A., Alsina, A., Diekmann, F., et al. (2015) Economic Analysis of the Treatment of End-Stage Renal Disease Treatment: Living-Donor Kidney Transplantation versus Hemodialysis. Transplantation Proceedings, 47, 3033. http://dx.doi.org/10.1016/j.transproceed.2014.12.005

[10] Howard, K., Salkeld, G., White, S., McDonald, S., Chadban, S., Craig, J.C. and Cass, A. (2009) The Cost-Effectiveness of Increasing Kidney Transplantation and Home-Based Dialysis. Nephrology (Carlton), 14, 123-132. http://dx.doi.org/10.1111/j.1440-1797.2008.01073.x

[11] Domínguez, J., Harrison, R. and Atal, R. (2011) Cost-Benefit Estimation of Cadaveric Kidney Transplantation: The Case of a Developing Country. Transplantation Proceedings, 43, 2300-2304. http://dx.doi.org/10.1016/j.transproceed.2011.06.006

[12] Rocha, M.J., Ferreira, S., Martins, L.S., Almeida, M., Dias, L., Pedroso, S., Henriques, A.C., Almeida, R. and Cabrita, A. (2012) Cost Analysis of Renal Replacement Therapy by Transplant in a System of Bundled Payment of Dialysis. Clinical Transplantation, 26, 529-531. http://dx.doi.org/10.1111/j.1399-0012.2011.01571.x

[13] Cavallo, M.C., Sepe, V., Conte, F., Abelli, M., Ticozzelli, E., Bottazzi, A. and Geraci, P.M. (2014) Cost-Effectiveness of Kidney Transplantation from DCD in Italy. Transplantation Proceedings, 46, 3289-3296. http://dx.doi.org/10.1016/j.transproceed.2014.09.146

[14] Just, P.M., Riella, M.C., Tschosik, E.A., Noe, L.L., Bhattacharyya, S.K. and de Charro, F. (2008) Economic Evaluations of Dialysis Treatment Modalities. Health Policy, 86, 163-180. http://dx.doi.org/10.1016/j.healthpol.2007.12.004

[15] Karopadi, A.N., Mason, G., Rettore, E. and Ronco, C. (2013) Cost of Peritoneal Dialysis and Haemodialysis across the World. Nephrology Dialysis Transplantation, 28, 2553-2569. http://dx.doi.org/10.1093/ndt/gft214 